This item was submitted to Loughborough's Research Repository by the author.

Items in Figshare are protected by copyright, with all rights reserved, unless otherwise indicated.

\title{
Adaptive wall-modelled large eddy simulation of jet noise in isolated and installed configurations
}

PLEASE CITE THE PUBLISHED VERSION

https://doi.org/10.2514/6.2018-3618

\section{PUBLISHER}

(c) American Institute of Aeronautics and Astronautics (AIAA)

\section{VERSION}

AM (Accepted Manuscript)

\section{PUBLISHER STATEMENT}

This work is made available according to the conditions of the Creative Commons Attribution-NonCommercialNoDerivatives 4.0 International (CC BY-NC-ND 4.0) licence. Full details of this licence are available at: https://creativecommons.org/licenses/by-nc-nd/4.0/

\section{LICENCE}

CC BY-NC-ND 4.0

\section{REPOSITORY RECORD}

Angelino, Matteo, Hao Xia, and Gary J. Page. 2018. "Adaptive Wall-modelled Large Eddy Simulation of Jet Noise in Isolated and Installed Configurations". figshare. https://hdl.handle.net/2134/33910. 


\title{
Adaptive Wall-Modelled Large Eddy Simulation of Jet Noise in Isolated and Installed Configurations
}

\author{
M. Angelino*, H. Xia ${ }^{\dagger}$ and G. J. Page ${ }^{\ddagger}$ \\ Department of Aeronautical and Automotive Engineering, Loughborough University \\ Loughborough, Leicestershire, LE11 3TU, United Kingdom
}

\begin{abstract}
The study of jet acoustics is crucial for future aeroengine designs. Although the highbypass ratio of modern turbofans can have a shielding effect on the core jet noise, there is an increased potential of interaction of the jet flow with wing and flap, and its effects on noise need to be thoroughly investigated. Wall-Modelled Large-Eddy Simulation (WMLES) is a powerful method to study the installation effects on jet noise, as it does not have strict near-wall requirements, allowing for a more uniform mesh for better noise propagation and a saving in computational cost. An adaptive wall model is here introduced and validated on channel flow, on the MD-30P/30N high-lift multi-element airfoil, and on the NASA High-Lift Common Research Model (HL-CRM). WMLES simulations, combined with the Ffowcs Williams and Hawkings (FW-H) sound extrapolation method, are performed on turbulent coaxial jets in isolated and installed configurations. Computed flow field and sound spectra present favourable agreement with experimental results, confirming key features of the installation effects on jet noise.
\end{abstract}

$a_{\infty} \quad$ speed of sound

Nomenclature

$D$ diameter

Ma Mach number

$\nu \quad$ viscosity

$\nu_{s g s}$ subgrid-scale viscosity

$\nu_{n w}$ near-wall turbulent viscosity

$\nu_{w} \quad$ wall turbulent viscosity

$p$ pressure

$r$ radial coordinate

St Strouhal number

$t$ physical time

$T$ temperature

$u$ velocity

$U_{b p}$ bypass stream axial velocity

$U_{c} \quad$ core stream axial velocity

$u_{\tau}$ friction velocity

$x \quad$ axial coordinate

$y \quad$ wall distance

$\varepsilon \quad$ upwinding parameter

$\theta$ polar angle

$\rho$ density

$\sigma \quad$ singular value of the velocity gradient tensor

$\tau_{w} \quad$ wall shear stress

*Research Associate, Rolls-Royce University Technology Centre

†Senior Lecturer, Rolls-Royce University Technology Centre

${ }^{\ddagger}$ Professor, Rolls-Royce University Technology Centre 


\section{Introduction}

The future of aviation is closely linked to aircraft environmental impact. Noise reduction is one of the industry's main concerns due to goals established by civil authorities, such as the $65 \%$ reduction of noise emissions by the year 2050 set by the European Commission. ${ }^{1}$ The noise from a propulsive jet is an important source at take-off and approach conditions even for modern high bypass ratio turbofans. Although the increase in bypass ratio can have a shielding effect on the core jet noise, there is an increased potential of interaction of the jet flow with the wing and the partially deployed high lift system, and its effects on noise need to be thoroughly investigated.

Since the pioneering work of Lighthill, ${ }^{2,3}$ numerous analytical and experimental studies ${ }^{4}$ have attempted to provide a more solid understanding of the jet noise generation mechanism. Recent advances in computational aeroacoustics ${ }^{5}$ have allowed to tackle the complexity of the phenomenon numerically under controlled conditions. Large-eddy simulations (LES) have proven to be a robust tool for prediction of the jet acoustic field. ${ }^{6-9}$ Relying on direct computation of the far-field noise is often not computationally affordable. Integral approaches, like Kirchhoff ${ }^{10}$ or Ffowcs Williams - Hawkings ${ }^{11}$ (FW-H), allow to evaluate the sound propagation from the perturbation field captured on a surface surrounding the jet, thus requiring an accurate solution only of the flow enclosed in the surface. The FW-H method has been validated in numerous studies ${ }^{12-16}$ and has been recently applied to jets from complex nozzles. ${ }^{17-19}$

Careful design of the grid is needed for noise propagation, with particular attention to ensure low growth rates and low aspect ratios. Depending on the complexity of the geometry, obtaining such a grid might not always be possible. Near-wall requirements for well-resolved LES are prohibitive, and even relying on hybrid approaches such as DES or hybrid RANS-LES usually means high aspect-ratios near the wall. Wall-modelled LES based on a wall-stress boundary condition (usually referred to as WMLES), can be a suitable alternative for the study of jet noise in complex configurations, as it has less strict near-wall requirements compared to wall-resolving methods, and is still able to capture the large eddies of the wing boundary layer ${ }^{20}$ the effects of which on jet noise need to be studied.

The aim of this work is to introduce an adaptive WMLES approach and to present its effectiveness in isolated and installed jet cases. The paper is structured as follows. Section II defines the numerical methods: the solvers, the adaptive WMLES, and the FW-H method. An extensive validation of the wall model is presented in Section III. Turbulent flow field and acoustic results for the isolated coaxial jet are discussed in Sections IV. Section V presents the setup and the analysis of the results for the installed case. Concluding remarks are provided in Section VI.

\section{Numerical methods}

\section{II.A. Solvers}

Two different solvers were used in order to assess the generality of implementation of the wall modelling. The jet cases and the HL-CRM case presented in this work were solved with Code-A, while the more fundamental cases studied for the validation of the wall modelling, i.e. the channel flow and the high-lift multi-element airfoil, were solved with Code-B. A brief description of the two solvers is given below.

\section{II.A.1. Code-A}

Code- $\mathrm{A}^{21}$ is a density-based cell-vertex finite volume industrial code used for turbomachinery design. The second order flux calculation is based on the Roe scheme

$$
F_{i j}=\frac{1}{2}\left(F\left(Q_{i}\right)+F\left(Q_{j}\right)\right)-\frac{1}{2} \varepsilon\left|A_{i j}\right|\left(L_{j}(Q)-L_{i}(Q)\right)
$$

where $A_{i j}$ is the Jacobian of the inviscid flux, $Q$ is the conserved variable vector, $L()$ is the pseudo-Laplacian operator and $\varepsilon$ is a tunable parameter to control the amount of upwinding. ${ }^{19}$ For the temporal discretisation, a standard three-stage Runge-Kutta explicit algorithm is employed. 


\section{II.A.2. Code- $B$}

Code-B is the OpenFOAM pressure-based compressible finite volume solver rhoPimpleFoam, built on a combination of the pressure-implicit split-operator (PISO) and the semi-implicit method for pressure-linked equations (SIMPLE). The SIMPLE sub-iterations (3 in the present work) allow a more stable convergence for larger time steps. For the convective terms a blending of a central differencing scheme and a second order upwind scheme is applied through a local factor $\varepsilon$, with the same rationale as in Code-A.

\section{II.B. Adaptive Wall-Modelled LES}

The Wall-Modelled LES presented in this work is based on the wall-stress modelling approach, in which the LES subgrid-scale model is defined all the way down to the wall, and the under-resolution of the boundary layer is compensated by imposing a wall-stress boundary condition.

\section{II.B.1. The subgrid-scale model}

The choice of the LES subgrid-scale (SGS) model is critical for jet applications. Standard models with constant coefficient are usually too dissipative in the initial free shear layer of the jet, thus delaying its transition. Dynamic models can overcome this issue ${ }^{22}$ at the price of stability, which is not always desirable when simulating complex flows. This is why implicit LES (or ILES) has been widely adopted in the literature, ${ }^{19,23,24}$ since the absence of an SGS model allows accurate capture of the jet behaviour, especially near the nozzle. An alternative LES approach ${ }^{25,26}$ can yield a more physical behaviour for boundary and free shear layers, by replacing the classical strain rate in the formulation of the subgrid-scale viscosity $\nu_{\text {sgs }}$, with other invariants of the velocity field. One of the latest examples of this approach, the $\sigma$-model, ${ }^{27}$ has been validated for jet noise, ${ }^{28,29}$ and chosen for the present work. The subgrid-scale viscosity is defined as

$$
\nu_{s g s}=\left(C_{m} \Delta\right)^{2} \mathcal{D}(\mathbf{u})
$$

with

$$
\mathcal{D}=\frac{\sigma_{3}\left(\sigma_{1}-\sigma_{2}\right)\left(\sigma_{2}-\sigma_{3}\right)}{\sigma_{1}^{2}}
$$

where $\sigma_{1} \geq \sigma_{2} \geq \sigma_{3} \geq 0$ are the three singular values of the velocity gradient tensor. Unlike the Smagorinsky model, the $\sigma$-model has, by construction, the property to automatically vanish as soon as the resolved field is either two-dimensional or two-component, including the pure shear and solid rotation cases. In addition, the model generates no subgrid-scale viscosity when the resolved scales are in pure axisymmetric or isotropic contraction/expansion. Finally, it has the appropriate cubic behaviour in the vicinity of solid boundaries without requiring any ad-hoc treatment. ${ }^{27}$ The robustness of the model makes it a good candidate for complex applications.

\section{II.B.2. The wall shear stress boundary condition}

Following Werner and Wengle, ${ }^{30}$ the wall boundary condition is specified by assuming that at the grid points closest to the wall, the instantaneous velocity $u$ is in phase with the instantaneous wall shear stress $\tau_{w}$ and the instantaneous velocity distribution is assumed to follow the law-of-the-wall. Here we choose to use Spalding's continuous formula for the law-of-the-wall ${ }^{31}$

$$
y^{+}=u^{+}+\frac{1}{E}\left(e^{\kappa u^{+}}-1-\kappa u^{+}-\frac{\left(\kappa u^{+}\right)^{2}}{2 !}-\frac{\left(\kappa u^{+}\right)^{3}}{3 !}\right)
$$

where $y^{+}=y u_{\tau} / \nu, u^{+}=u / u_{\tau}, \kappa=0.41$ and $E=9.8$. The formula is solved iteratively for $u_{\tau}$ through the Newton-Raphson method. The resulting required wall shear stress

$$
\tau_{w}=\rho \cdot u_{\tau}^{2}
$$

is applied by defining the wall turbulent viscosity

$$
\nu_{w}=\frac{\tau_{w}}{\rho \cdot \partial u / \partial y}-\nu
$$




\section{II.B.3. The near-wall correction}

This wall-stress function needs to be balanced by an appropriate internal field in the immediate proximity of the wall. Werner and Wengle ${ }^{30}$ replaced the Smagorinsky length scale with the minimum value between the Prantdl mixing length $\kappa y$ and a corrected LES filter width. Shur et al. ${ }^{32}$ adopted a similar approach for a Hybrid RANS-LES with the Smagorinsky model in fully-resolved boundary layers, with the addition of the wall-damping function by Piomelli ${ }^{33}\left(1-e^{-\left(y^{+} / A^{+}\right)^{3}}\right)$. In both these studies a near-wall treatment was necessary due to the high levels of the Smagorinsky SGS viscosity near the wall resulting in excessive turbulence damping.

In the present work, the grid is assumed to be only mildly anisotropic near the wall, and therefore suitable for LES with no need for a hybrid RANS-LES blending zone. The under-resolution of the boundary layer, on the other hand, means that the subgrid-scale viscosity near the wall needs to be corrected, similarly to the above-mentioned studies. In this case this is necessary because the SGS viscosity obtained with the $\sigma$-model

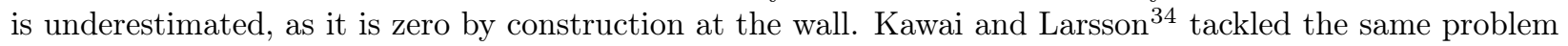
for the dynamic Smagorinsky model by using one-sided formulas for the derivatives and by extrapolating from the interior nodes the model coefficient. Here we choose a less complex approach, by adjusting the near-wall turbulent viscosity (similarly to Templeton et al. ${ }^{35}$ ) in a RANS fashion:

$$
\nu_{n w}=(\kappa y)^{2} \cdot\left(1-e^{-\left(y^{+} / A^{+}\right)^{3}}\right) \cdot S
$$

with $S=\sqrt{2 S_{i j} S_{i j}}, \kappa=0.41$ and $A^{+}=25$. This near-wall formulation is similar to the Baldwin-Lomax RANS model, ${ }^{36}$ which has been widely used in aerospace and turbomachinery applications, thanks to its suitability for high-speed flows with thin attached boundary-layers.

This near-wall correction is necessary only in the first cell/node from the wall, since the LES model can quickly resume the LES behaviour in the adjacent layers. It is also an adaptive correction that can be used seamlessly with any resolution of the boundary layer (provided that the grid is relatively isotropic and the modelling only involves the inner boundary layer), since it vanishes with a physical behaviour when the boundary layer is fully resolved. This allows for a simple and robust implementation, with no additional parameters controlling the RANS-LES blending, as is required in DES or typical Hybrid RANS-LES models.

A schematic of the use of $\nu_{s g s}, \nu_{n w}$ and $\nu_{w}$ in the cell-vertex solver and in the cell-centred solver is reported in Figure 1.
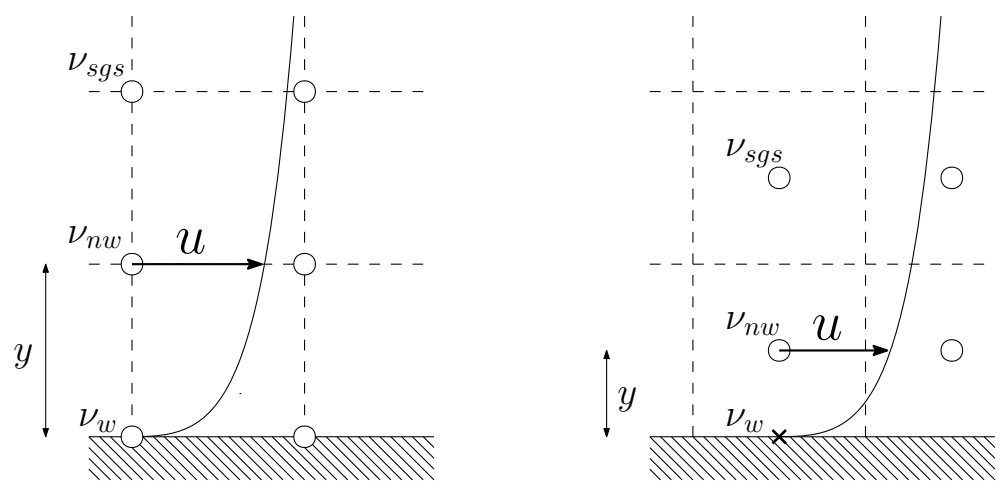

Figure 1. Schematic of the implementation of the wall modelling for the cell-vertex solver (left) and the cell-centred solver (right). 


\section{II.C. Far-field acoustic computation}

For the far-field noise, the $\mathrm{FW}-\mathrm{H}$ surface integral ${ }^{37}$ is computed. This yields the far-field acoustic pressure fluctuation $p^{\prime}(\mathbf{x}, t)$. Since the noise source is inside the surface (if the surface is large enough and far enough from the jet exit region), a simplification can be made by omitting the volume quadrupole integral. This, as suggested by Shur et al. ${ }^{38}$ and Di Francescantonio, ${ }^{37}$ saves substantial data storage. The integral equation is as follows:

$$
4 \pi p^{\prime}=\frac{\partial}{\partial t} \int_{S}\left[\frac{\rho u_{n}}{r}\right] d S+\frac{1}{a_{\infty}} \frac{\partial}{\partial t} \int_{S}\left[\frac{p_{n r}^{\prime}+\rho u_{n} u_{r}}{r}\right] d S+\int_{S}\left[\frac{p_{n r}^{\prime}+\rho u_{n} u_{r}}{r^{2}}\right] d S
$$

In the above, $\mathbf{r}$ ( $r$ being its modulus) defines the observer position, $a_{\infty}$ stands for the ambient speed of sound and $S$ is the FW-H surface. The quantities in the square brackets are computed at "retarded" times. Also, $n_{j}$ is the component of the unit outward normal vector on the surface, and $u_{j}$ is the velocity component. Surface data is stored while the simulation is performed, ready for later post-processing.

\section{Wall modelling validation}

\section{III.A. Channel flow}

A channel flow simulation with $\mathrm{Re}_{\tau}=2000$ was performed for a first validation of the present wall modelling. In Figure 2 the model yields the correct law-of-the-wall behaviour for both a well-resolved $\left(y^{+}=3\right)$ and an under-resolved $\left(y^{+}=25\right)$ boundary layer in channel flow, with a slight mismatch in the log-layer, typical of this kind of models. ${ }^{39}$ The adaptive nature of this model makes it suitable for complex configurations, in which the near-wall refinement might change drastically within the same domain. Similarly to the channel case, the correct under-resolved estimation of the wall-shear stress is expected to be beneficial for the prediction of the flow profile inside the jet nozzle, and therefore for the overall evolution of the jet.

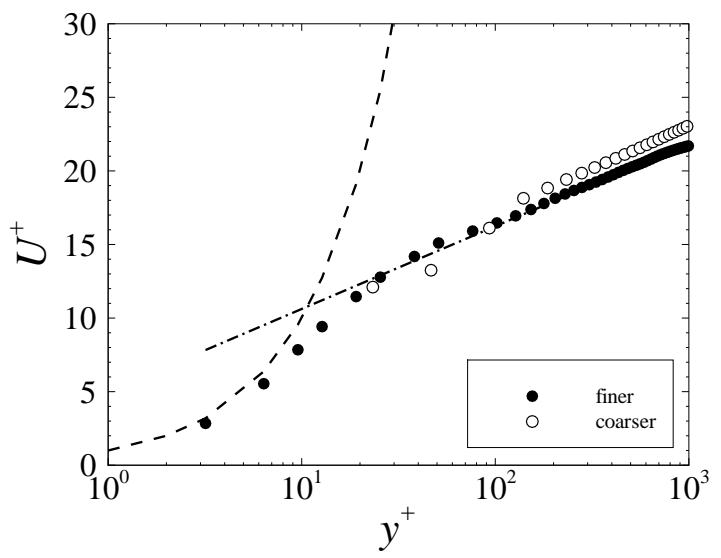

Figure 2. Channel flow velocity profile with the adaptive Wall-Modelled LES

\section{III.B. High-lift multi-element airfoil}

Further validation of the wall modelling was conducted to test its suitability to capture the lift in an installed jet configuration. Simulations were performed on the multi-element high-lift airfoil MD-30P/30N, at two angles of attack, 8 and 19 degrees, at which the flow is attached and the wall model is therefore fully reliable. The angles of attack were intended to be close to a take-off and to an approach condition, which are of interest for jet noise applications. The grid is fully structured and has 12 million cells, with a spanwise domain width of $4 \%$ of the chord, and a near-wall resolution around $y^{+}=100$. Figure 3 shows the instantaneous flow field around the airfoil at $\mathrm{AoA}=19^{\circ}$. The accurate capture of the turbulent features of the flow along the flap might play an important role in the study of jet-flap interaction. 
In Figure 4 (left) the surface pressure coefficient compares well with experiments. ${ }^{40}$ Figure 4 (right) shows the correct prediction of lift, compared to the case with no wall modelling, which clearly yields an overprediction. A series of contributions presented in a recent study on slat noise ${ }^{41}$ (many of which were performed on a $70 \mathrm{M}$ grid with wall-resolved DES) is reported in the same figure with the median value of the entire set of simulations, and an error bar indicating the confidence interval. This comparison confirms that our wall model has the potential to yield similar results at a lower computational cost.

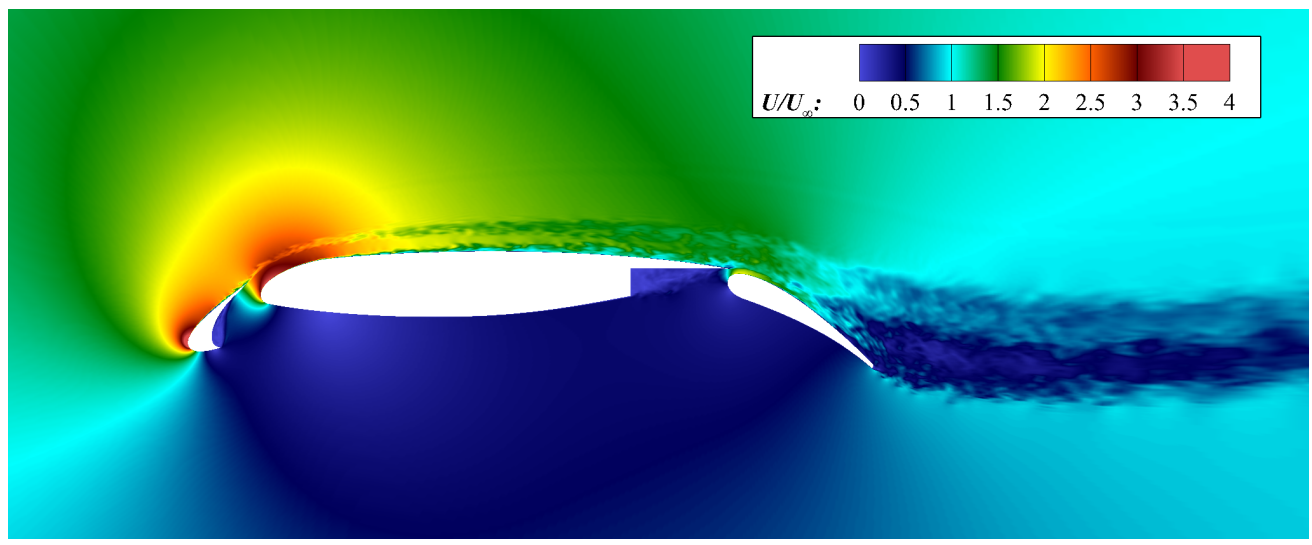

Figure 3. Instantaneous velocity magnitude around a MD-30P/30N high-lift airfoil with deployed slat and flap $\left(\operatorname{AoA}=19^{\circ}\right)$.
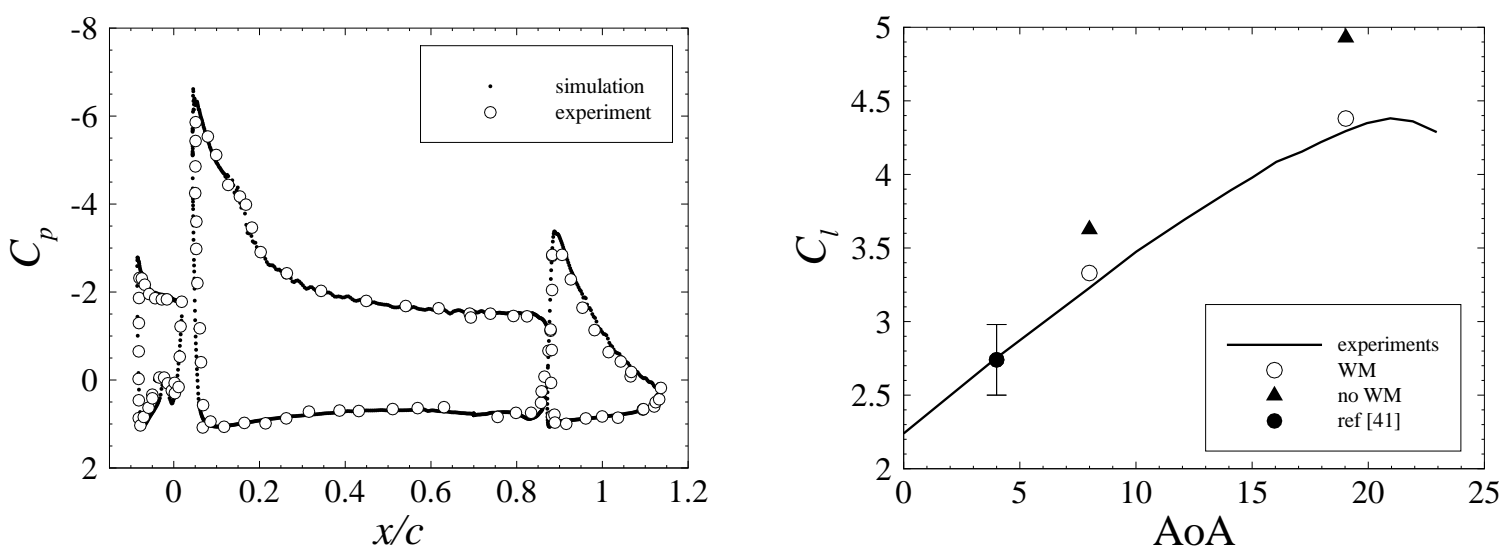

Figure 4. MD-30P $/ 30 \mathrm{~N}$ surface pressure coefficient $\left(\mathrm{AoA}=8^{\circ}\right)$ and lift curve.

\section{III.C. High-lift Common Research Model (HL-CRM)}

The final test case for the the wall model validation was chosen to be a full 3D aircraft, based on the NASA High-Lift Common Research Model (HL-CRM) in landing configuration. ${ }^{42}$ Table 1 provides details about the geometry and the operating conditions. The desired Reynolds number of 3.26 million, based on the mean aerodynamic chord (MAC), is achieved with the full scale geometry by scaling up the viscosity appropriately.

Table 1. HL-CRM geometric parameters and operating conditions.

\begin{tabular}{cccc}
\hline $\mathrm{Ma}$ & $\mathrm{AoA}$ & $\mathrm{MAC}$ & $\mathrm{Re}_{\mathrm{MAC}}$ \\
\hline 0.2 & $16^{\circ}$ & $7 \mathrm{~m}$ & $3.26 \mathrm{M}$ \\
\hline
\end{tabular}


The simulation was performed on a hybrid structured-unstructured mesh of $50 \mathrm{M}$ cells, with near-wall hexahedral layers. In order for the grid to be suitable for the present WMLES, the resolution at the walls was set to $y^{+}<100$, and $\Delta x^{+}, \Delta z^{+}=300-3000$.

The Q-criterion of Figure 5 reveals the wing-tip and flap-tip vortices, as well as very fine turbulent structures captured by the LES model on the wing. Figure 6 shows a very good agreement between the pressure coefficient from the present case and that obtained ${ }^{42}$ with a much finer wall-resolved RANS simulation. Due to the under-resolution of the boundary layer, the results obtained with the current approach are not expected to be as accurate as a wall-resolved method. Nonetheless, the possibility of predicting essential features, such as the surface pressure distribution, with a computationally efficient eddy-resolving simulation, at a cost comparable to wall-resolving RANS methods, can be crucial for installed jet noise applications.

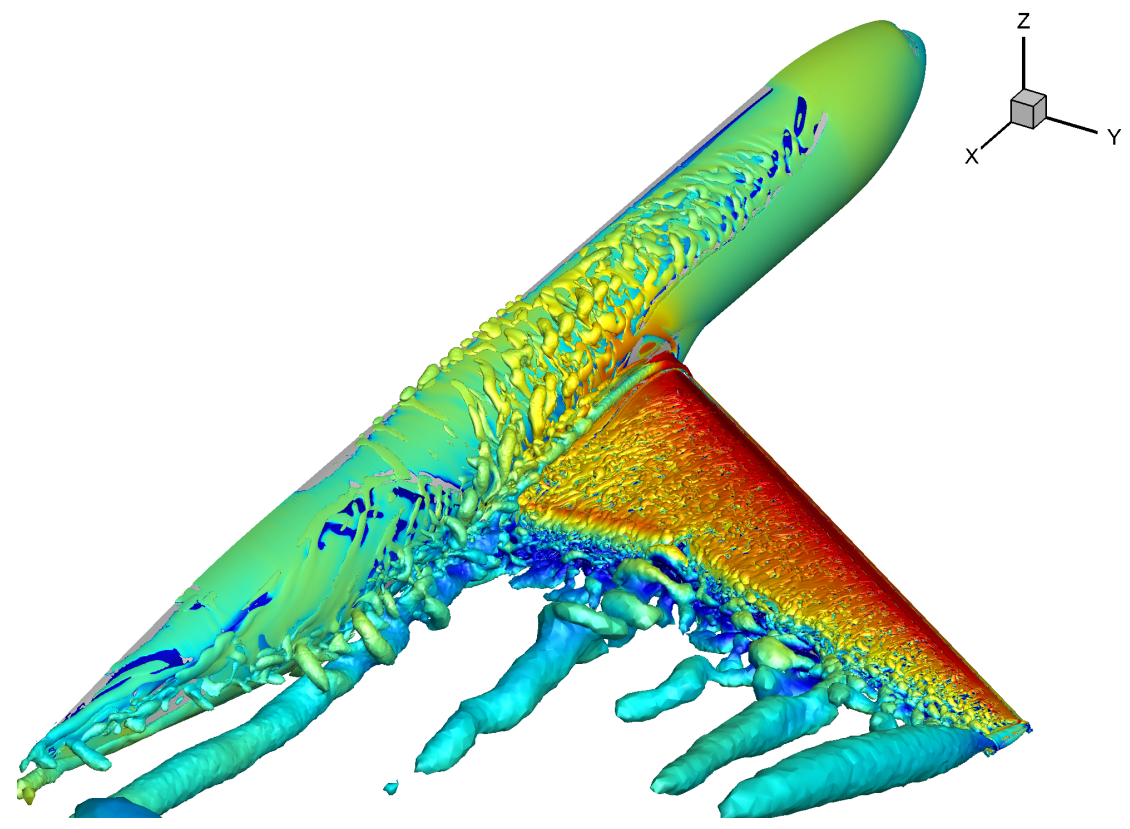

Figure 5. Q-criterion of the HL-CRM, coloured by streamwise velocity.
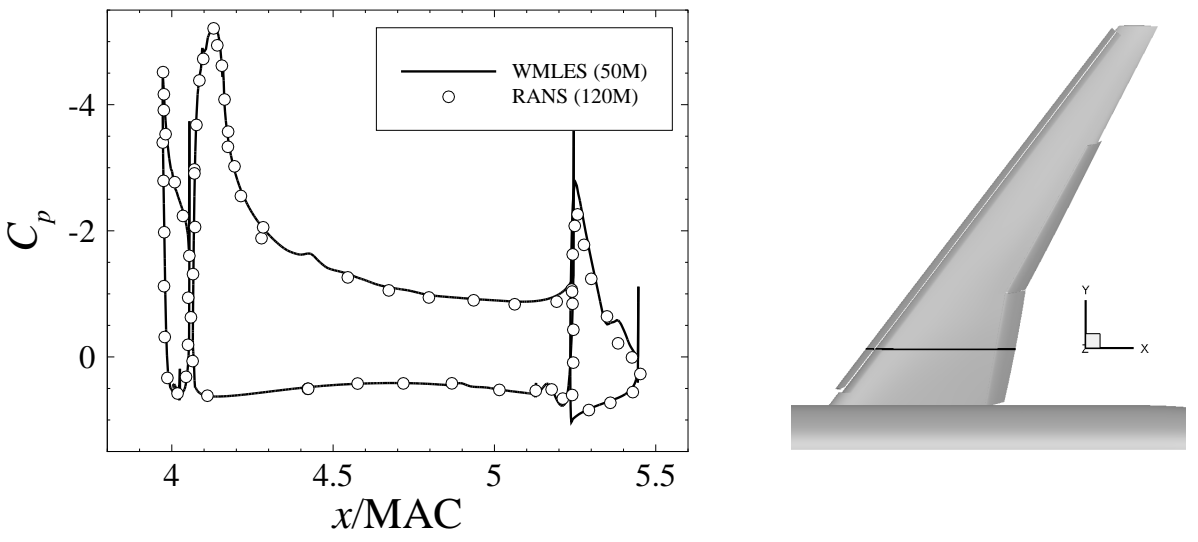

Figure 6. HL-CRM surface pressure coefficient (left) at spanwise location $y=1$ MAC (right). 


\section{Isolated coaxial jet}

\section{IV.A. Case setup}

The case chosen as baseline for the isolated coaxial jet is that of the CoJeN project with no flight-stream (see Table 2).

Table 2. CoJeN operating conditions.

\begin{tabular}{ccccc}
\hline$U_{c}$ & $U_{b p}$ & $\mathrm{Ma}_{c}$ & $\mathrm{Ma}_{b p}$ & $\mathrm{TR}$ \\
\hline 480 & 306 & 0.86 & 0.9 & 2.7 \\
\hline
\end{tabular}

The cylindrical computational domain ranges from $x / D_{b p}=-2$ to 100 , and from $r / D_{b p}=20$ to 40 . The $50 \mathrm{M}$ grid is completely structured, with a central block connected to the plug (see Figure 7), and a near-wall resolution around $y^{+}=50$. The spatial numerical scheme uses a smoothing parameter $\varepsilon=0.5$, which is considered a good compromise in terms of reducing the dissipation while avoiding spurious sound waves. $\varepsilon=1$ is used as a sponge zone near the far-field boundaries. The simulation was run using explicit time stepping ensuring $\mathrm{CFL}<0.5$. A transient of $300 t^{*}$ (where the non-dimensional time $t^{*}=D_{b p} / U_{c}$ ) was necessary to initialize the flow, and another $50 t^{*}$ was used for flow statistics and FW-H.

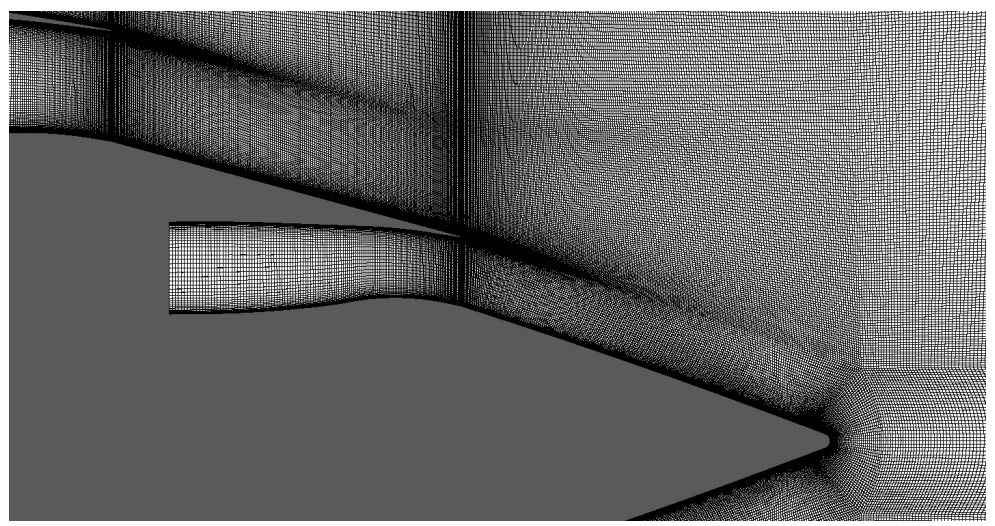

Figure 7. Grid detail near the CoJeN nozzle.

\section{IV.B. Instantaneous and mean flow}

Figure 8 shows a snapshot of the jet instantaneous flow field of the isolated CoJeN case. The velocity contours (rainbow color map) reveal the shielding effect of the bypass, with a delayed shear layer development of the (red) jet core. The greyscale pressure contours qualitatively locate very strong sound sources near the nozzle lips, and identify a preferred directions of propagation at low polar angles $\left(\theta \approx 30^{\circ}\right)$, as expected.

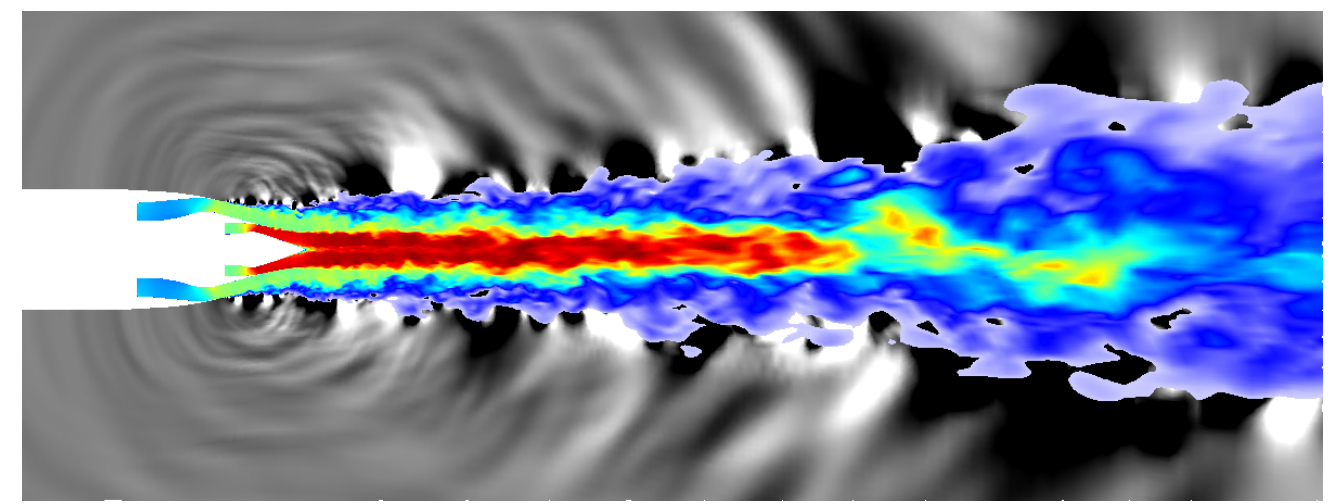

Figure 8. Near-field acoustic wave dilatation visualized by pressure contours (greyscale) and velocity contours (rainbow) for the CoJeN case. 
Centerline statistics (Figure 9) agree well with experiments, thanks to the careful meshing of the plug region and to the effective modelling of turbulence of the $\sigma$-model. The axial velocity rises rapidly downstream of the plug, reaching a peak at $x / D_{b p} \approx 3.5$, and the subsequent rate of decay is well captured. The normal stress shows the same agreement, with its maximum peak matching the measurement further downstream at $x / D_{b p} \approx 6$.
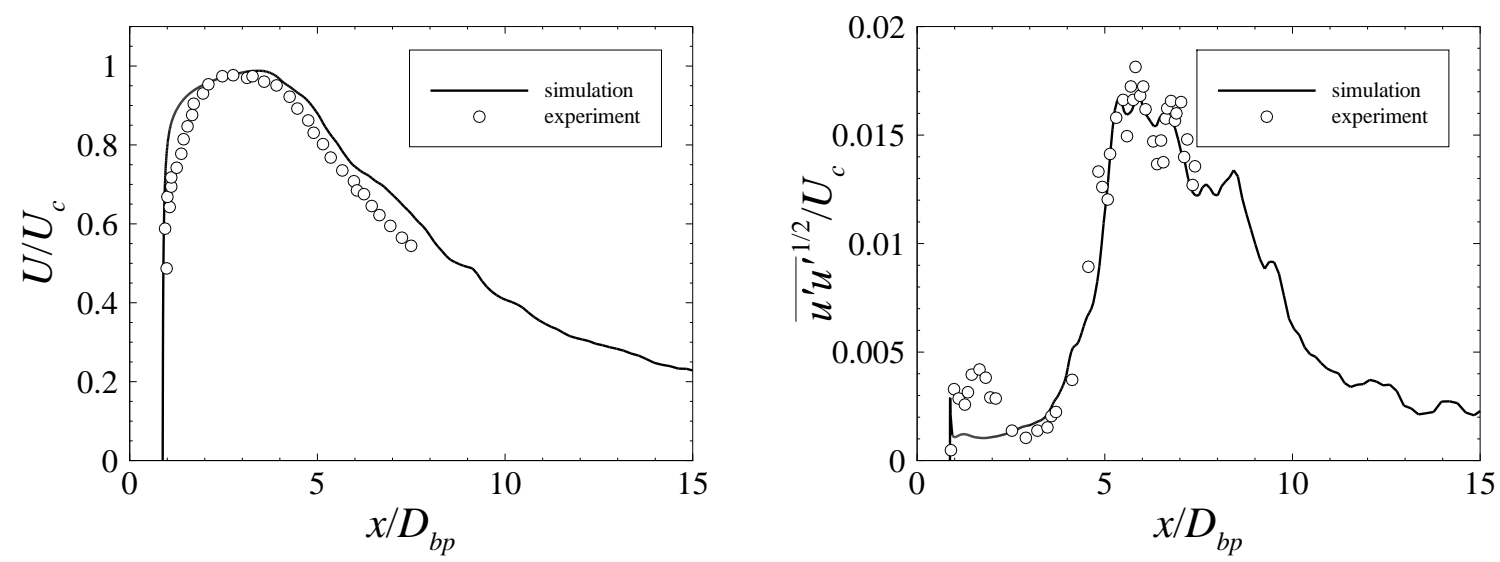

Figure 9. Axial velocity and normal stress along the centerline.

\section{IV.C. Acoustics}

Figure 10 shows a sketch of the FW-H surface with the sound observer positions. The placement of the FW-H surface was chosen to avoid hydrodynamic pressure fluctuations, while still remaining as close as possible to the jet. The position of the closing disk at 30D is a good compromise, as it is far enough not to introduce spurious results, and close enough to keep the initialization time to a minimum.

The acoustic results are azimuthally averaged and Figure 11 shows the PSD for two different polar angles $\theta=30^{\circ}$ and $120^{\circ}$. The agreement with experiments ${ }^{43}$ is very good, with a cut-off frequency of St $\approx 3-4$. The peak noise level and the decay rate at high frequencies are well captured at every polar angle.

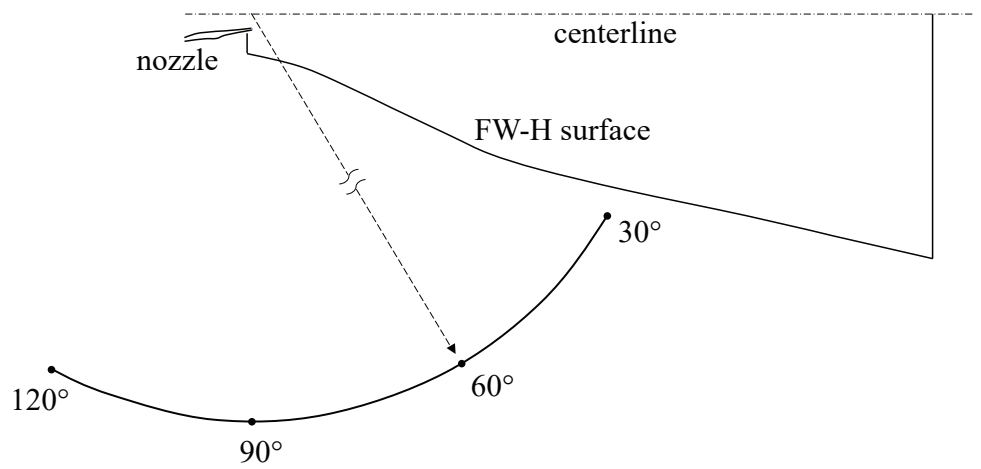

Figure 10. Representation of the FW-H surface profile and the far-field sound observer position. 

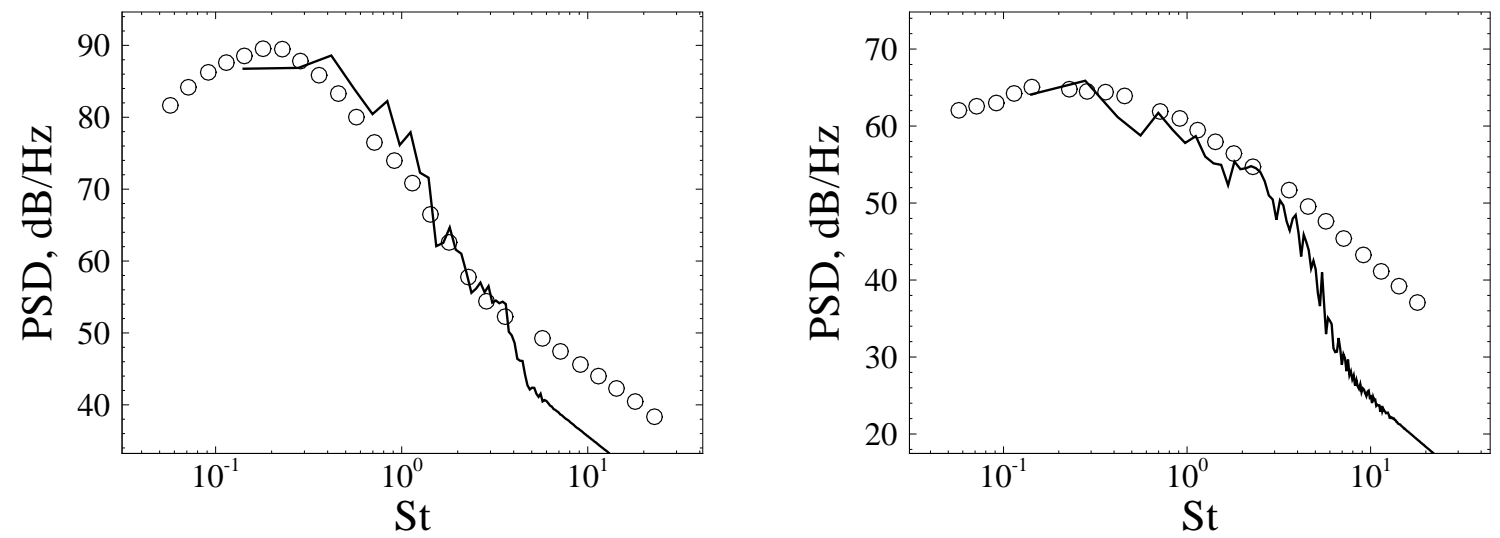

Figure 11. Far-field sound PSD at $\theta=30^{\circ}$ (left) and $120^{\circ}$ (right) for the coaxial isolated jet (symbols expt; solid line LES).

\section{Installed coaxial jet}

\section{V.A. Case setup}

The installed case consists of a simplified transport aircraft geometry, with fuselage, one wing with flaps deployed, and the coaxial engine nozzle with pylon. Geometry and experimental data were produced under the SYMPHONY project. The flow conditions are representative of the range of conditions encountered by modern aeroengines in post-takeoff. For comparison with experimental results obtained with an open-jet wind tunnel in a large anechoic chamber, the simulated jet flow is supplied via a feed pipe (see Figure 12). The computational domain ranges from $x / D_{b p}=-30$ to 100 in the axial direction, and from $r / D_{b p}=30$ to 50 in the radial direction. The $120 \mathrm{M}$ grid is almost completely structured in the areas of interest (blue in Figure 12), consisting of $80 \mathrm{M}$ hexahedra, almost equally divided between the jet region and the airframe. The rest of the domain (green) is unstructured and allows for a more rapid coarsening towards the far-field. The near-wall resolution is around $y^{+}=70$ on the nozzle boundaries, and $y^{+}<100$ on the wing. As can be seen in Figure 13, a particular effort has been made to ensure low growth rates and low aspect ratios in the jet region, for an optimal noise propagation.

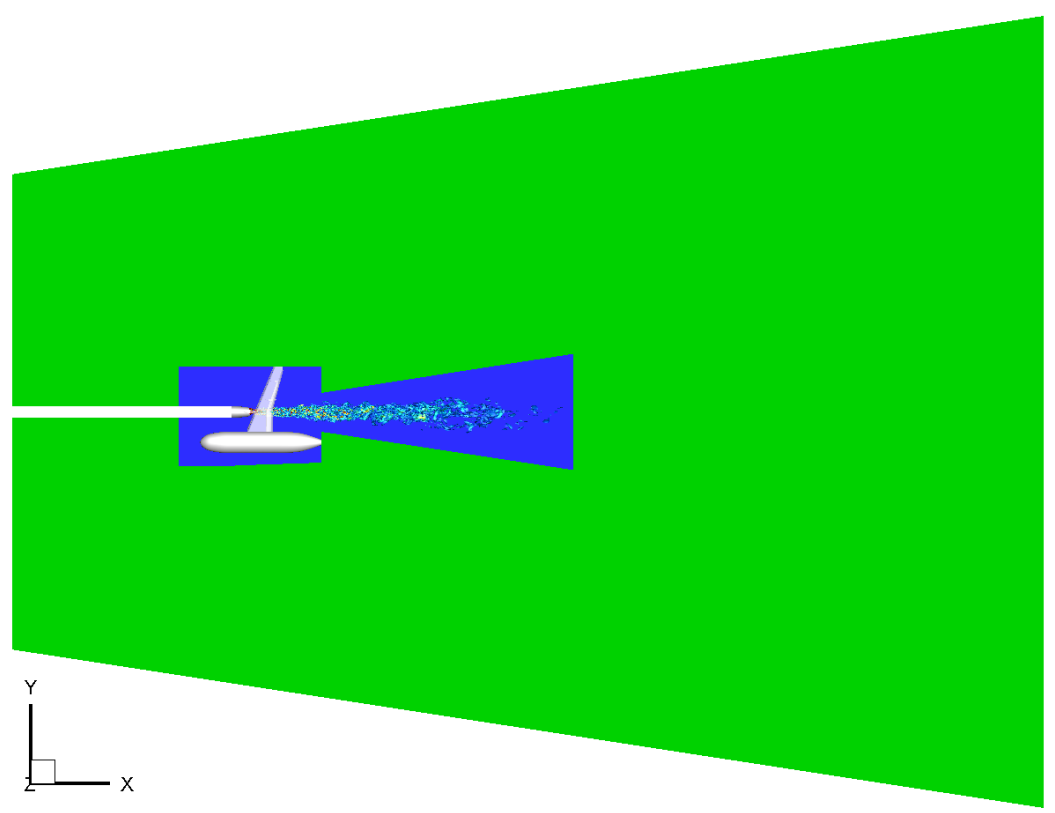

Figure 12. Schematic of the structured (blue) and unstructured (green) regions of the installed case grid. 


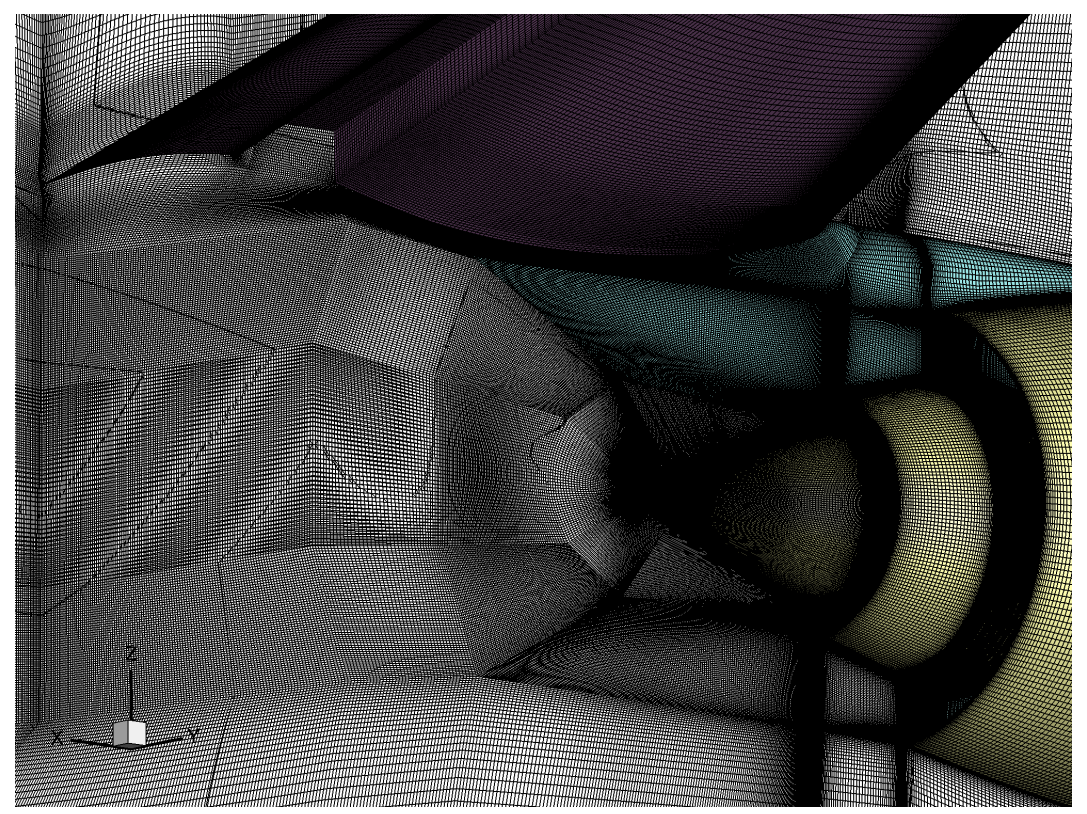

Figure 13. Close-up of the installed case grid near the nozzle (magenta - wing; cyan - pylon; yellow - nozzle).

The spatial numerical scheme is the same as the isolated case, with $\varepsilon=0.5$ (and $\varepsilon=1$ near the far-field boundaries). The simulation was run using explicit time stepping ensuring $\mathrm{CFL}<0.5$. A transient of $200 t^{*}$ (where the non-dimensional time $t^{*}=D_{b p} / U_{c}$ ) was necessary to initialize the flow, and another $100 t^{*}$ was used for flow statistics and FW-H.

\section{V.B. Instantaneous and mean flow}

Figure 14 shows the jet Q-criterion, and acoustic waves on a cut-plane immediately below the engine. It can be noticed how the sound propagation is well captured in the inner structured region of the mesh, whereas strong dissipation occurs away from the inner region.

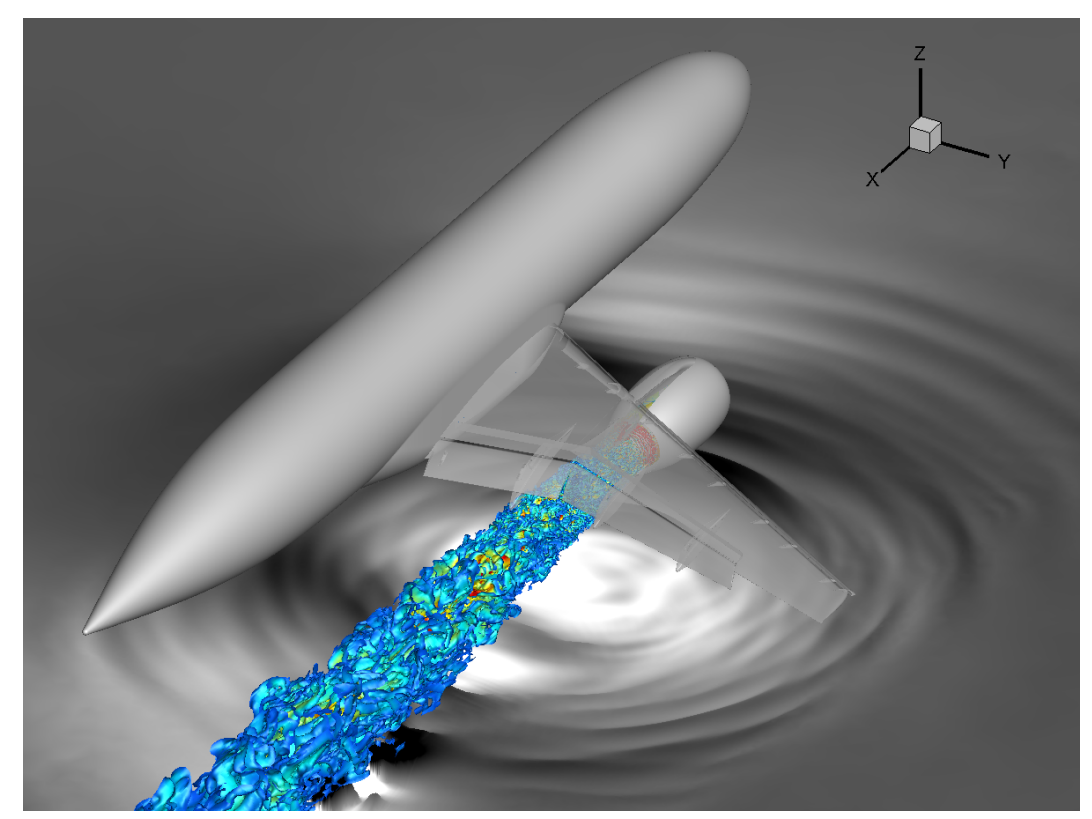

Figure 14. Q-criterion coloured by axial velocity (rainbow), and pressure waves at $z / D_{b p}=-0.2$ (greyscale). The solid surfaces are only representative of the actual geometry. 
Centerline statistics are reported in Figure 15. No experimental comparison is available at this stage, but the overall behaviour is similar to the isolated case. The drop in axial velocity at $x / D_{b p} \approx 2.5$, right after its peak at $x / D_{b p} \approx 2$, is thought to be due to the presence of the pylon. The normal stress presents the expected peak at $x / D_{b p} \approx 7$, corresponding to the collapse of the potential core.
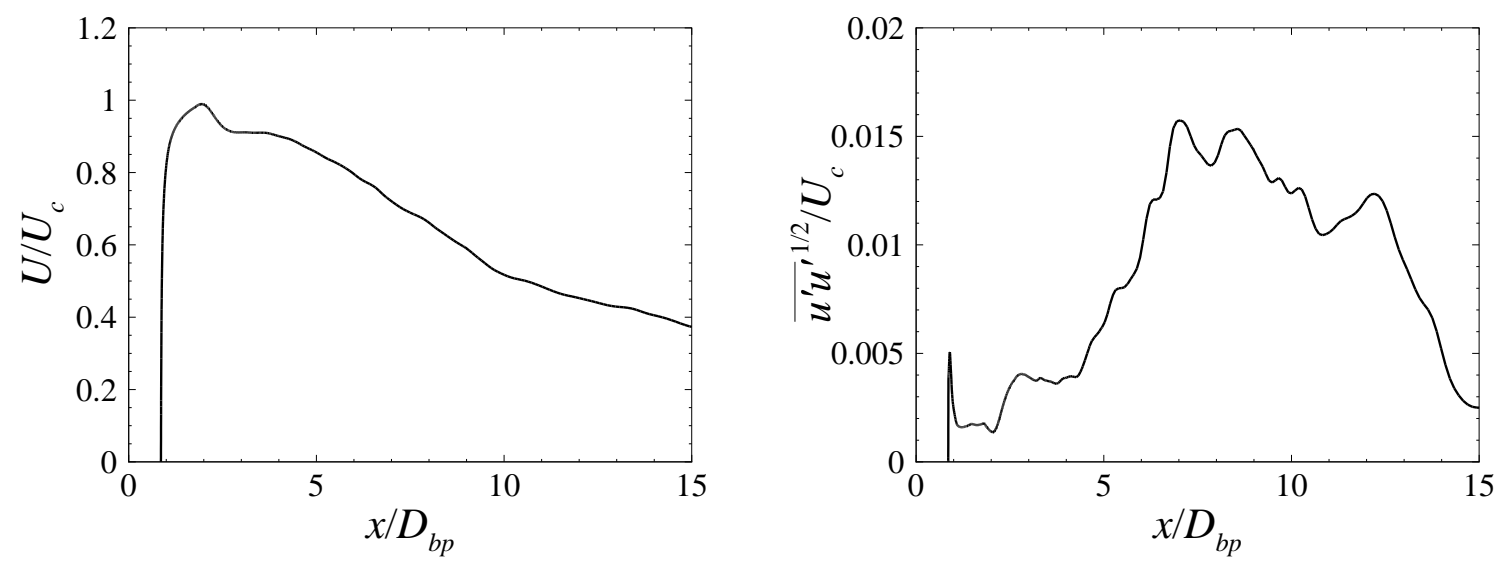

Figure 15. Axial velocity and normal stress along the centerline for the installed case.

\section{V.C. Acoustics}

The interaction of the acoustic waves with wing and flap is illustrated in Figure 16, displaying $d p / d t$ contours in the $x z$ cut-plane. The expected noise source where the jet interacts with the flap is clearly noticeable, together with the high-frequency scattering from the wing leading edge.

The FW-H surface was determined in order to include these sources. As can be seen in Figure 17, near the solid boundaries the surface consists of a series of elliptical sections encasing the wing. Further downstream it resumes a circular section similar to the isolated case. The radial distance from the jet was chosen to avoid hydrodynamic noise sources, while still remaining in the structured region of the grid. The position of the closing disk at 40D is a good compromise for flight-stream conditions, as it is far enough not to introduce spurious results, and close enough to keep the initialization time to a minimum.

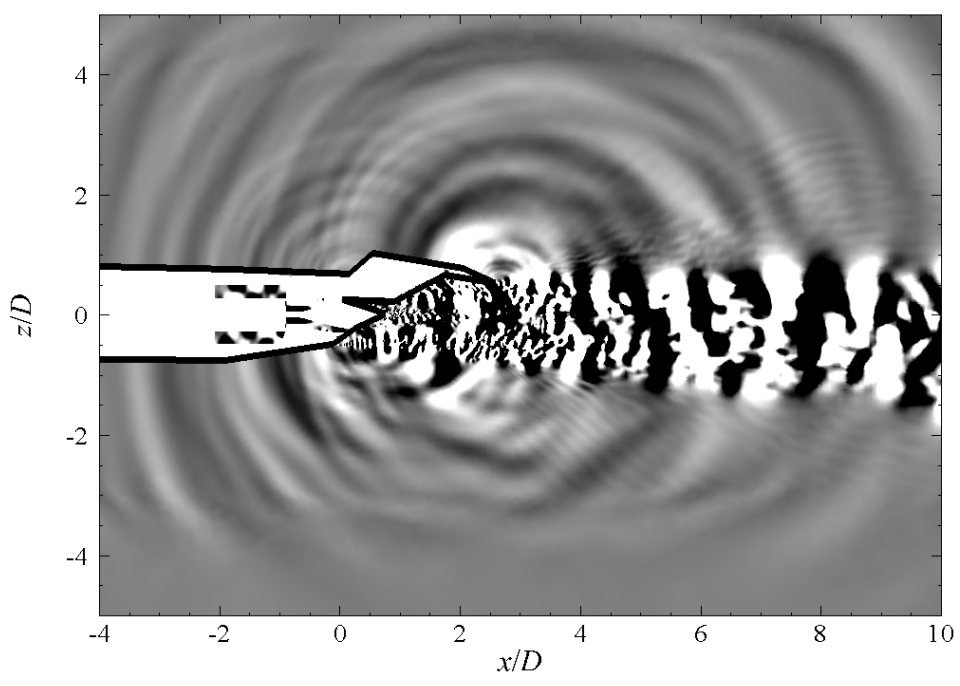

Figure 16. Instantaneous $d p / d t$ contours for the installed case 


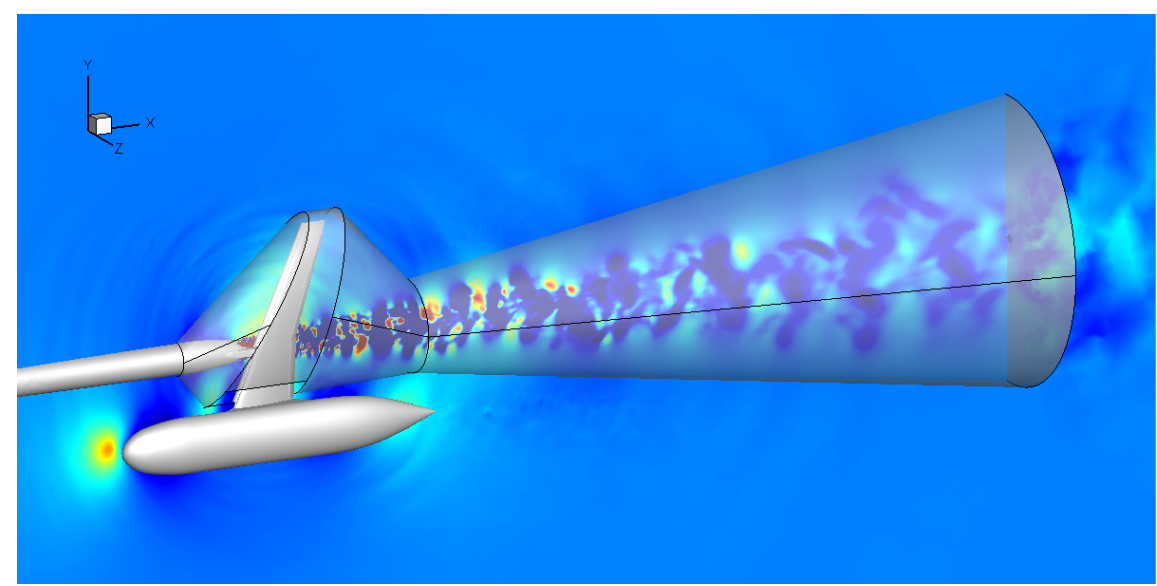

Figure 17. FWH surface (transparent) and pressure contours on the $x y$ plane. The solid boundaries are only representative of the actual geometry.

The acoustic results are shown in Figure 18 for flyover polar angles $\theta=90^{\circ}$ and $120^{\circ}$, where $0^{\circ}$ corresponds to the direction of the jet, $90^{\circ}$ is the downward direction from the engine, and $120^{\circ}$ is slightly tilted towards the flight direction (see Figure 10). Since the azimuthal average is not possible in this case, the PSD is filtered by averaging over one-third octave bands.

The matching low-frequency drop of the spectra is a sign that the placement of the FW-H surface is appropriate. The peak noise levels are in agreement with the experiments, as are the decay rates before the cut-off frequency $(\mathrm{St} \approx 2.5)$. The interaction of the sound waves with the wing and the flap produces an increase of the jet noise directed downwards especially at lower frequencies, compared to the isolated case. The double peak at $120^{\circ}$, properly captured by the simulation, is evidence of this effect, and suggests that installation effects have a stronger influence in the upstream direction.
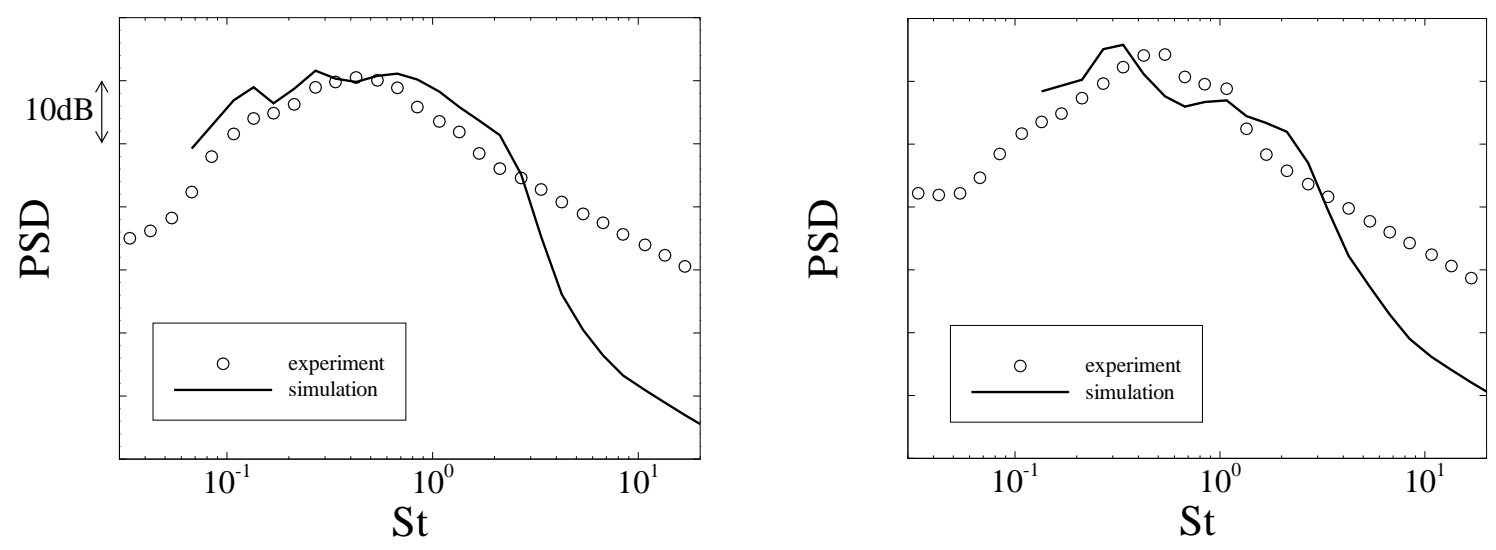

Figure 18. Far-field sound PSD at $\theta=90^{\circ}$ (left) and $120^{\circ}$ (right) for the installed case.

\section{Conclusions}

A robust and adaptive WMLES method has been presented. Validation of the model was successfully conducted on channel flow, on a high-lift multi-element airfoil, and on the NASA HL-CRM, showing its computational efficiency compared to wall-resolving methods. The adaptive nature of this model makes it suitable for complex geometries, in which the near-wall refinement might change drastically within the same domain, and for jet noise studies, in which more uniform grids can improve sound propagation.

Simulations of turbulent coaxial jets in isolated and installed configurations show very good agreement with experimental data, for both near-field fluctuating quantities and far-field sound spectra. An analysis of 
the sound propagation in the installed case reveals an additional noise source due to the jet-flap interaction, and a high-frequency scattering from the wing leading edge. The flyover installation effects show a strong influence in the spectral content, particularly at upstream polar angles.

The present work can serve as a starting point for future studies to build a more comprehensive understanding of the installation effects on jet noise.

\section{Acknowledgments}

The work on the isolated co-axial jet was conducted under the Innovate UK SILOET II project 13 (reference number 113013), in collaboration with Rolls-Royce plc. The geometry was produced under the EU 6th Framework Project CoJeN, contract number AST3-CT-2003-502790.

The installed case is part of the ongoing Innovate UK ACAPELLA project (reference number 113086), in collaboration with Rolls-Royce plc. Geometry and experimental data were produced under the Innovate UK SYMPHONY project (reference number 100539) and provided by Rolls-Royce plc.

The authors acknowledge the support from the UK Turbulence Consortium (UKTC), under grant number EP/L000261. CPU time and IT support from ARCHER, HPC Midlands and Loughborough University are greatly appreciated.

\section{References} 2011.

${ }^{1}$ High Level Group on Aviation Research, "Flightpath 2050 Europe's Vision for Aviation," tech. rep., European Commission,

${ }^{2}$ M. J. Lighthill, "On Sound Generated Aerodynamically. I. General Theory," Proceedings of the Royal Society A: Mathematical, Physical and Engineering Sciences, vol. 211, pp. 564-587, Mar. 1952.

${ }^{3}$ M. J. Lighthill, "On Sound Generated Aerodynamically. II. Turbulence as a Source of Sound," Proceedings of the Royal Society A: Mathematical, Physical and Engineering Sciences, vol. 222, pp. 1-32, Feb. 1954.

${ }^{4}$ C. K. Tam, "Jet Noise: Since 1952," Theoretical and Computational Fluid Dynamics, vol. 10, pp. 393-405, Jan. 1998.

${ }^{5}$ D. J. Bodony and S. K. Lele, "Current Status of Jet Noise Predictions Using Large-Eddy Simulation," AIAA Journal, vol. 46, pp. 364-380, Feb. 2008.

${ }^{6}$ C. Bogey, C. Bailly, and D. Juvé, "Noise Investigation of a High Subsonic, Moderate Reynolds Number Jet Using a Compressible Large Eddy Simulation," Theoretical and Computational Fluid Dynamics, vol. 16, pp. 273-297, Mar. 2003.

${ }^{7}$ D. J. Bodony and S. K. Lele, "On using large-eddy simulation for the prediction of noise from cold and heated turbulent jets," Physics of Fluids, vol. 17, no. 8, p. 085103, 2005.

${ }^{8} \mathrm{C}$. Bogey and C. Bailly, "Computation of a high Reynolds number jet and its radiated noise using large eddy simulation based on explicit filtering," Computers \& Fluids, vol. 35, pp. 1344-1358, Dec. 2006.

${ }^{9}$ C. Bogey, S. Barré, D. Juvé, and C. Bailly, "Simulation of a hot coaxial jet: Direct noise prediction and flow-acoustics correlations," Physics of Fluids, vol. 21, no. 3, p. 035105, 2009.

${ }^{10}$ G. Kirchhoff, "Zur Theorie der Lichtstrahlen," Annalen der Physik, vol. 254, no. 4, pp. 663-695, 1883.

${ }^{11}$ J. E. FFowcs Williams and D. L. Hawkings, "Sound Generation by Turbulence and Surfaces in Arbitrary Motion," Philosophical Transactions of the Royal Society A: Mathematical, Physical and Engineering Sciences, vol. 264, pp. 321-342, May 1969.

${ }^{12}$ A. Biancherin, N. Lupoglazoff, G. Rahier, and F. Vuillot, "Comprehensive 3d Unsteady Simulations of Subsonic and Supersonic Hot Jet Flow-Fields: Part 2: Acoustic Analysis," AIAA 2002-2600, June 2002.

${ }^{13}$ P. J. Morris, L. N. Long, T. E. Scheidegger, and S. Boluriaan, "Simulations of Supersonic Jet Noise," International Journal of Aeroacoustics, vol. 1, pp. 17-41, Jan. 2002.

${ }^{14}$ A. Uzun, A. Lyrintzis, and G. Blaisdell, "Coupling of Integral Acoustics Methods with LES for Jet Noise Prediction," AIAA 2004-0517, Jan. 2004.

${ }^{15}$ P. R. Spalart and M. L. Shur, "Variants of the Ffowcs Williams - Hawkings Equation and Their Coupling with Simulations of Hot Jets," International Journal of Aeroacoustics, vol. 8, pp. 477-491, July 2009.

${ }^{16}$ S. Mendez, M. Shoeybi, S. Lele, and P. Moin, "On the use of the Ffowcs Williams-Hawkings equation to predict far-field jet noise from large-eddy simulations," International Journal of Aeroacoustics, vol. 12, pp. 1-20, June 2013.

${ }^{17}$ M. Shur, P. Spalart, and M. Strelets, "Noise prediction for increasingly complex jets. Part II: Applications," International Journal of Aeroacoustics, vol. 4, pp. 247-266, July 2005.

${ }^{18}$ M. Shur, P. Spalart, and M. Strelets, "Noise prediction for increasingly complex jets. Part I: Methods and tests," International Journal of Aeroacoustics, vol. 4, pp. 213-246, July 2005.

${ }^{19}$ H. Xia, P. G. Tucker, and S. Eastwood, "Large-eddy simulations of chevron jet flows with noise predictions," International Journal of Heat and Fluid Flow, vol. 30, pp. 1067-1079, Dec. 2009.

${ }^{20} \mathrm{~J}$. Bodart and J. Larsson, "Wall-modeled large eddy simulation in complex geometries with application to high-lift devices," in Annual Research Briefs, (Center for Turbulence Research), pp. 37-48, 2011.

${ }^{21} \mathrm{P}$. Moinier, Algorithm developments for an unstructured viscous flow solver. PhD thesis, University of Oxford, 1999.

${ }^{22}$ M. Angelino, A. Boghi, and F. Gori, "Numerical solution of three-dimensional rectangular submerged jets with the evidence of the undisturbed region of flow," Numerical Heat Transfer, Part A: Applications, vol. 70, pp. 815-830, Oct. 2016. 
${ }^{23}$ F. F. Grinstein and C. Fureby, "Recent Progress on MILES for High Reynolds Number Flows," Journal of Fluids Engineering, vol. 124, no. 4, p. 848, 2002.

${ }^{24}$ P. G. Tucker, "Novel MILES computations for jet flows and noise," International Journal of Heat and Fluid Flow, vol. 25, pp. 625-635, Aug. 2004.

${ }^{25}$ F. Nicoud and F. Ducros, "Subgrid-Scale Stress Modelling Based on the Square of the Velocity Gradient Tensor," Flow, Turbulence and Combustion, vol. 62, no. 3, pp. 183-200, 1999.

${ }^{26}$ A. W. Vreman, "An eddy-viscosity subgrid-scale model for turbulent shear flow: Algebraic theory and applications," Physics of Fluids, vol. 16, pp. 3670-3681, Oct. 2004.

${ }^{27}$ F. Nicoud, H. B. Toda, O. Cabrit, S. Bose, and J. Lee, "Using singular values to build a subgrid-scale model for large eddy simulations," Physics of Fluids, vol. 23, no. 8, p. 085106, 2011.

${ }^{28}$ M. Angelino, H. Xia, M. A. Moratilla-Vega, and G. J. Page, "Far-field Noise Prediction of Round and Serrated Jets with Increasingly Refined Grids," AIAA 2016-3047, (Lyon, France), May 2016.

${ }^{29}$ M. Angelino, M. A. Moratilla-Vega, H. Xia, and G. J. Page, "Large-eddy simulations of high Reynolds number jets with a suitable subgrid-scale model for solver dependency study," in 11th International ERCOFTAC Symposium on Engineering Turbulence Modelling and Measurements, (Palermo, Italy), Sept. 2016.

${ }^{30} \mathrm{H}$. Werner and H. Wengle, "Large-Eddy Simulation of Turbulent Flow Over and Around a Cube in a Plate Channel," in Turbulent Shear Flows 8, pp. 155-168, Berlin, Heidelberg: Springer Berlin Heidelberg, 1993.

${ }^{31}$ D. B. Spalding, "A Single Formula for the "Law of the Wall"," Journal of Applied Mechanics, vol. 28, no. 3, p. $455,1961$.

${ }^{32}$ M. L. Shur, P. R. Spalart, M. K. Strelets, and A. K. Travin, "A hybrid RANS-LES approach with delayed-DES and wall-modelled LES capabilities," International Journal of Heat and Fluid Flow, vol. 29, pp. 1638-1649, Dec. 2008.

${ }^{33}$ U. Piomelli, "High Reynolds number calculations using the dynamic subgrid-scale stress model," Physics of Fluids A: Fluid Dynamics, vol. 5, pp. 1484-1490, June 1993.

${ }^{34}$ S. Kawai and J. Larsson, "Wall-modeling in large eddy simulation: Length scales, grid resolution, and accuracy," Physics of Fluids, vol. 24, p. 015105, Jan. 2012.

${ }^{35}$ J. A. Templeton, G. Medic, and G. Kalitzin, "An eddy-viscosity based near-wall treatment for coarse grid large-eddy simulation," Physics of Fluids, vol. 17, no. 10, p. 105101, 2005.

${ }^{36}$ B. Baldwin and H. Lomax, "Thin-layer approximation and algebraic model for separated turbulentflows," AIAA 19780257, Jan. 1978.

${ }^{37}$ P. Di Francescantonio, "A new boundary integral formulation for the prediction of sound radiation," Journal of Sound and Vibration, vol. 202, pp. 491-509, May 1997.

${ }^{38}$ M. Shur, P. Spalart, M. Strelets, and A. Travin, "Towards the prediction of noise from jet engines," International Journal of Heat and Fluid Flow, vol. 24, pp. 551-561, Aug. 2003.

${ }^{39}$ X. I. A. Yang, G. I. Park, and P. Moin, "Log-layer mismatch and modeling of the fluctuating wall stress in wall-modeled large-eddy simulations," Physical Review Fluids, vol. 2, Oct. 2017.

${ }^{40}$ V. Chin, D. Peters, F. Spaid, and R. Mcghee, "Flowfield measurements about a multi-element airfoil at high Reynolds numbers," AIAA 1993-3137, July 1993.

${ }^{41}$ M. M. Choudhari and D. P. Lockard, "Assessment of Slat Noise Predictions for 30p30n High-Lift Configuration from BANC-III Workshop," AIAA 2015-2844, June 2015.

${ }^{42}$ C. L. Rumsey, J. P. Slotnick, and A. J. Sclafani, "Overview and Summary of the Third AIAA High Lift Prediction Workshop," American Institute of Aeronautics and Astronautics, Jan. 2018.

${ }^{43}$ C. J. Mead, C. Wrighton, and K. Britchford, "An Experimental Study of Coaxial Jets Using Acoustic PIV and LDA Methods (CoJeN)," AIAA 2015-3122, June 2015. 